\title{
Relación entre factores administrativos e innovación
}

José Antonio Varela Loyola Universidad Politécnica de Tlaxcala joseantonio.varela@uptlax.edu.mx

José Nemorio Méndez Mendoza Universidad Politécnica de Tlaxcala josenemorio.mendez@uptlax.edu.mx

DOI: https://doi.org/10.21158/01208160.n83.2017.1826

Fecha de recepción: 22 de febrero de 2017

Fecha de aprobación: 12 de junio de 2017

Cómo citar este artículo / To reference this article / Comment citer cet article / Para citar este artigo:

Varela, J. A. y Méndez, J. N. (2017). Relación entre factores administrativos e innovación. Revista EAN, 83, pp. 31-50. https:// doi.org/10.21158/01208160.n83.2017.1826

\section{Resumen}

El objetivo del artículo es determinar la relación existente entre la innovación técnica e innovación administrativa en organizaciones conocidas como pequeñas y medianas empresas (PyMEs) del ramo de las autopartes, considerando aquellos factores que promueven la innovación: planteamiento de objetivos, enfoque de mercado, estrategia competitiva, mercado global y relación con el cliente. Para ello, se realizó un estudio de correlación de rho Spearman asociando las variables de innovación técnica y administrativa con 12 PyMEs del ramo de las autopartes ubicadas en el estado de Tlaxcala - México-. Los resultados de esta investigación evidencian una relación positiva entre el planteamiento de objetivos, el enfoque de mercado, la estrategia competitiva, el mercado global y la relación con el cliente con la innovación técnica, mientras que no hay evidencia de una relación entre todos los factores con la innovación administrativa.

\section{Palabras clave}

Innovación técnica, innovación administrativa, PyMEs, factores administrativos.

\footnotetext{
${ }^{1}$ Ingeniero Industrial, Maestro en Administración y Doctor en Planeación Estratégica y Dirección de Tecnología; y profesor Investigador de la Universidad Politécnica de Tlaxcala, México. ORCID: http://orcid.org/0000-0002-4154-3170

${ }^{2}$ Ingeniero Industrial, Maestro en Ciencias de la Ingeniería Industrial y profesor Investigador de la Universidad Politécnica de Tlaxcala, México. ORCID: http://orcid.org/0000-0002-1991-9987
} 


\section{Relationship between administrative factors and innovation}

Abstract. This article aims at determining the existing relation between technical and administrative innovation in well-known small and medium- size companies (PyMEs) from the auto parts sector, taking into account those factors which promote innovation: stating aims, focusing on marketing, competitive strategy, global market and customer relation. For this reason, a correlation study of Rho Spearman was made linking technical and administrative innovation in twelve PyMEs from the auto parts sector located in Tlaxcala, Mexico. Its research outcomes show the positive relation between aims, marketing focus, competitive strategy, global market and the relation of customers with technical innovation, while there are no evidences of an existing relation between these factors and administrative innovation.

Key words. Technical innovation, administrative innovation, SMEs, administrative factors.

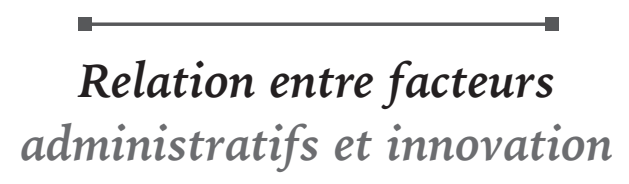

Résumé. L'objectif de cet article consisteà déterminer la relation existante entrel'innovation techniqueet l'innovation administrative dans les petites et moyennes entreprises (PME) du secteur des pièces détachées automobiles. Les facteurs permettant l'innovation dans ce secteur d'activité sont la mise en place d'objectifs spécifiques, une stratégie compétitive, un marché global et la relation client. Une étude de corrélation de rho Spearman a par ailleurs été réalisée associant les variables d'innovation technique et administrative de douze PME du secteur des pièces détachées automobiles de l'état de Tlaxcala, au Mexique. Les résultats de l'investigation mettent en évidence une relation positive entre les facteurs d'innovation cités plus haut et l'innovation technique même s'il n'existe pas d'évidence d'une relation entre tous les facteurs et l'innovation administrative.

Mots clefs. Innovation technique, innovation administrative, PME, facteurs administratifs.

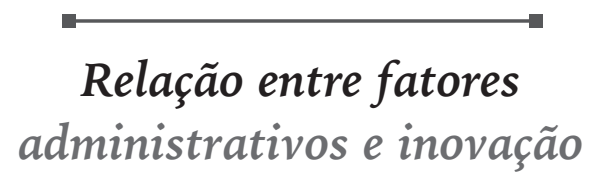

Resumo. O objetivo do artigo é determinar a relação entre inovação técnica e inovação administrativa em organizações conhecidas como pequenas e médias empresas (PMEs) no setor de autopeças, considerando os fatores que promovem a inovação: propostas de objetivos, foco no mercado, estratégia competitiva, mercado global e relacionamento com o cliente. Para isso, foi realizado um estudo de correlação de rho Spearman, associando as variáveis de inovação técnica e administrativa com doze PMEs do ramo de autopeças localizadas no estado de Tlaxcala (México). Os resultados desta pesquisa mostram uma relação positiva entre a proposta de de objetivos, o foco no mercado, a estratégia competitiva, o mercado global e a relação do cliente com a inovação técnica, enquanto que não há evidência de relação entre todos os fatores com inovação administrativa.

Palavras-chave. Inovação técnica, inovação administrativa, PMEs, fatores administrativos. 


\section{Introducción}

Q n un entorno cada vez más competiEtivo y cambiante, la innovación en las empresas ha pasado de ser un elemento de diferenciación a una exigencia para la supervivencia. En los últimos tiempos, la innovación ha constituido un elemento clave para la ventaja competitiva en las organizaciones (Afuah, 199; Bowen, Rostami y Steel, 2010). Últimamente, el fenómeno de la innovación ha recibido atención en la investigación científica, puesto que es factor clave para cambios significativos en el seno de las organizaciones (Damanpour y Wischnevsky, 2006; López, Montes, Prieto y Vázquez, 2008), y como tal es considerada esencial para el sostenimiento y la competitividad de las empresas en el ámbito global (Chan Kin y Mauborgne, 2004; Foxona, Grossa, Chaseb, Howesb, Arnallc y Anderson, 2005).

Por su parte, Hull y Tidd (2003) mencionan que debe existir una mayor atención para estudiar el fenómeno de la innovación por parte de los investigadores y así explicar cómo sucede la innovación en las empresas y la relación que existe con los factores administrativos. Sin embargo, pocos estudios se centran en investigar cuáles factores de índole administrativo contribuyen en la generación de la innovación (Donate y Guadamillas, 2008; Lee y Kelley, 2008).

La literatura señala diversos factores como determinantes para la generación de innovación, factores que son tanto internos como externos (Berson, Oreg y Dvir, 2008; Lin, 2006). De los factores que generan innovación, se incluyen: la cultura de apoyo (Martensen, Dahlgaard, Park-Dahlgaard y Grønholdt, 2007; Skarzynski y Gibson, 2008; Smith, Ball y Van der Meer, 2008), las prácticas de liderazgo (Martensen,
Dahlgaard, Park-Dahlgaard y Grønholdt, 2007; Saunila y Ukko, 2014; Smith, Busi, Ball y Van der Meer, 2008), los procesos y las herramientas para la gestión de ideas (Skarzynski y Gibson, 2008) y las fuentes externas de información (Laforet, 2008), habiendo pocas investigaciones que estudien sobre los factores administrativos que forman parte de los procesos operacionales de las empresas.

Entre las investigaciones que estudian aquellos factores administrativos para incentivar la innovación, se encuentra el realizado por Simon (2009), quien menciona que existe una serie de ejes que ayudan a las empresas a innovar, tales como los objetivos organizacionales, el mercado, la estrategia competitiva, la globalización, el cliente, la estructura organizacional y el liderazgo.

Otros estudios mencionan que la generación de la innovación se inicia a partir de una serie de factores administrativos que las empresas están ejecutando de forma continua (Daft, 2007; Suñe, Bravo, Mundet y Herrera, 2012), y que los factores contienen una serie de indicadores (Yamakawa y Ostos, 2011), aunque en estos estudios no hacen mención sobre cuáles son los factores y cuáles son los indicadores. Mientras que Adams, Bessant y Phelps (2006) y Ernst (2002) estudiaron que la innovación se genera a partir de un conjunto de elementos administrativos que se ejecutan en las organizaciones y que el éxito o fracaso depende de la forma como es la ejecución en las empresas. Varela y Flores (2014) proponen cinco factores administrativos que promueven la innovación. La limitación que tienen estos estudios es que no se realizó una comprobación empírica para determinar la relación existente, ya que estos se centran en investigaciones teóricas. 
En México, se han realizado investigaciones sobre la innovación en las empresas del ramo de las autopartes. Por ejemplo, González y Martin (2013) concluyeron que la innovación es fundamental para que las empresas que están bajo entornos económicos desfavorables aumenten la competitividad, y como tal la innovación debe ayudar a ser más competitivo. También mencionan que la innovación es usada como una estrategia para hacer frente a la existencia de factores internos y externos que condicionan el sector automotor en México y que en momentos de crisis desempeña un papel primordial para salir de ella (Rivas y Flores, 2007).

La industria automotriz exige a los proveedores mayor eficiencia y productividad, por tanto, deben mejorar los procesos de producción y distribución; a este tipo de mejoras se les conoce como innovaciones técnicas (Vicencio, 2007).

Por su parte, Cevilla (2005) menciona que para la industria de autopartes en América Latina es fundamental aprender a realizar innovaciones técnicas, puesto que este tipo de innovación es primordial por la exigencia del sector automotor y es considerada como el principio para la mejora continua, muy necesaria en el sector de autopartes, por lo que las empresas se ven en la necesidad de establecer metodologías de mejora continua para iniciar la innovación.

Existe otro tipo de mejoras que se centra en la organización de la empresa, a las funciones a este tipo de mejora se les puede llamar innovación administrativa. Es de llamar la atención que esta no es estudiada en México y en América Latina por parte de los investigadores, sino que las fuentes consultadas se centran solo en la innovación técnica.

El objetivo de este trabajo de investigación es analizar si existe una relación entre los factores administrativos y la generación de innovación técnica e innovación administrativa, planteándose la siguiente pregunta: ¿los factores administrativos tienen relación con la generación de innovación técnica e innovación administrativa en las PyMEs del sector de autopartes del estado de Tlaxcala, México? Y si es así, definir los rasgos característicos de este tipo de PyMEs con respecto a la innovación, de tal forma que si se evidencia que existe una relación entre estos factores, sean adoptados por aquellas empresas que estén interesadas en incentivar la innovación en sus operaciones.

Para dar respuesta a la pregunta de investigación, el trabajo se desarrolla de la siguiente manera. En la primera parte, se hace una revisión de la literatura para definir el concepto de innovación y detectar aquellos factores administrativos que fomentan la innovación en las empresas, con el fin de formular las hipótesis de investigación. La segunda parte está constituida por la descripción de la metodología que se ha de utilizar. En la tercera, se discuten los resultados donde se dan respuesta a las hipótesis planteadas. Finalmente, se proponen las recomendaciones, se identifican las limitaciones de la investigación y se sugieren temas de investigación. 


\section{Marco conceptual}

A través de la innovación las organizaAciones se adaptan, se diversifican e incluso se rejuvenecen o reinventan para adecuarse a las condiciones cambiantes de la tecnología y del mercado.

La innovación es la generación y adopción de una nueva idea que se tiene que convertir en un producto o servicio que deseen los clientes. Incluso, es una nueva forma de hacer cosas: es mediante la introducción de una técnica o proceso de producción que no precisa fundarse en un descubrimiento nuevo desde el punto de vista científico, sino que se puede adoptar algo ya existente y adaptarlo o en algunos casos mejorarlo (Manual de Oslo, 2007; Porter, 1990). Las empresas innovadoras suelen ser más flexibles, tienen mayor capacidad de adaptación y de respuesta a los cambios y logran explotar las oportunidades existentes en mayor medida que la competencia; esto les permite lograr una ventaja competitiva sostenible mediante la implementación de nuevos procesos, nuevos productos o nuevos enfoques de administración para incrementar la eficiencia (Kotler y Caslione, 2010).

En sí, se dice que existe innovación técnica cuando en las empresas hay una mejora en procesos de producción, en métodos de trabajo, en la tecnología empleada y en el diseño del producto, mientras que para decir que hay innovación administrativa en una empresa debe haber mejoras en la organización, en las funciones de trabajo, cambios en los puestos de trabajo y mejoras en el modelo de negocios (Hamel, 2012; Manual de Oslo, 2007, Varela y Flores, 2014).
Damanpour, Szabat y Evan (1989) sustentan que para mantener o mejorar el nivel de desempeño, la adopción balanceada de una innovación técnica y una innovación administrativa es más efectiva en la organización que la implementación de solo una de ellas. Ademá, Vahs (2003) recomienda que si existe alguna innovación en el producto es recomendable hacer cambios en los procesos y en la estructura organizacional, ya que estos ayudarán a la adaptación de las innovaciones, la reducción de costo y acrecentar la calidad. Las innovaciones técnicas promueven la efectividad organizacional, mientras que las innovaciones administrativas son necesarias para obtener un balance entre la estructura social y el sistema técnico de la organización.

Las innovaciones técnicas y administrativas se inician en diferentes partes de la organización y son diversos factores que las motivan (Damanpour, 1996). En muchas empresas el problema no es la generación de ideas para la innovación, sino los factores que ayuden a desarrollar esas ideas y concretarlas (Cash, Earl y Morison, 2008). Se considera que una organización innovadora requiere una estructura orgánica flexible y no estandarizada para lograr innovaciones sofisticadas, y como tal, que la empresa tenga ciertos factores en la administración que ayuden a impulsar la innovación (Daft, 2007; Kotler y Caslione, 2010). Existe cierto tipo de empresas que tienen ventajas de comportamiento en factores administrativos que les permiten reaccionar de manera más oportuna y flexible ante cambios externos, y a partir de ello generar innovación (Kotler y Caslione, 2010) (Tabla 1). 
Tabla 1. Indicadores de innovación técnica e innovación administrativa.

\begin{tabular}{|c|c|}
\hline Innovación técnica & Innovación administrativa \\
\hline $\begin{array}{l}\text { - Mejora en el proceso de producción (IT1) } \\
\text { - Mejora en los métodos de trabajo (IT 2) } \\
\text { - Mejora en la tecnología empleada (IT3) } \\
\text { - Mejora en el diseño del producto (IT4) }\end{array}$ & $\begin{array}{l}\text { - Mejora en la organización del trabajo (IA1) } \\
\text { - Mejora en las funciones de trabajo (IA2) } \\
\text { - Cambios en los puestos de trabajo (IA3) } \\
\text { - Mejora en el modelo de negocios (IA4) }\end{array}$ \\
\hline
\end{tabular}

Fuente. Elaboración propia.

\subsection{Factores administrativos que promueven la innovación}

De acuerdo con la revisión documental sobre los factores administrativos que promueven la innovación, Varela y Flores (2014) mencionan que en las investigaciones sobre factores que promueven la innovación existe una diversidad de criterios sobre cómo es la creación de innovación en las empresas. Los estudios han tratado de identificar aquellas actividades clave del proceso de la innovación, refieren que la innovación es generada a partir del contexto orientado a la tecnología; otras investigaciones se centran en que la innovación se genera a partir de actividades organizacionales, y unas más señalan que es importante medir la cantidad de inversiones realizadas por las empresas y la generación de nuevos productos (Adams, Bessant y Phelps, 2006).

Para Cooper y Kleinschmidt (1995), influyen cinco factores para la generación de innovación: el proceso de desarrollo de nuevos productos, la estrategia, la organización, la cultura y la gestión del compromiso. Este modelo pasa por alto la innovación en contextos no técnicos y otros factores importantes, como el papel

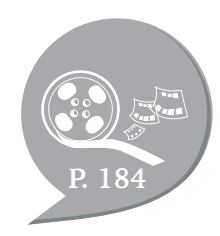
del conocimiento.
Verhaeghe y Kfir (2002) mencionan que la importancia en el proceso de innovación se centra en la forma en cómo se transfiriere la tecnología, en cómo es la generación de ideas y en la ejecución de la comercialización.

Cormican y O'Sullivan (2004) conciben la gestión de la innovación de productos como un proceso continuo y transversal, lo cual genera la participación y la integración de un número cada vez mayor de las diferentes competencias dentro de la organización. Este proceso requiere una adopción con éxito y la adaptación de un sistema sociotécnico para todos los aspectos de la organización, que incluye a las personas y el proceso.

Adams, Bessant y Phelps (2006) indican otra serie de factores que se ejecutan en el proceso intermedio en generación de innovación y que se deben medir para decir que una empresa innova. Los factores consisten en categorías tales como la gestión del conocimiento, la estrategia, la organización, la cultura y la comercialización. Por su parte, Simon (2009) encuentra que existen un grupo de empresas que realiza una gestión adecuada en la innovación. Durante el estudio se detectaron factores específicos orientados a los objetivos organizacionales, el mercado, la estrategia competitiva, la globalización, el cliente, las fortalezas, la estructura organizacional y el liderazgo. 
Como se describió en párrafos anteriores, cada una de las investigaciones ponen factores administrativos que generan innovación combinados con factores técnicos y financieros. Sin embargo, no existe una investigación que haya conjuntado solo los factores administrativos, de ahí la importancia de estudiarlos e investigar cómo es el comportamiento en las PyMEs.

En la revisión realizada por Varela y Flores (2014), concluyen que son cinco los factores administrativos que promueven la innovación (Figura 1).

Figura 1. Factores administrativos con indicadores clave.
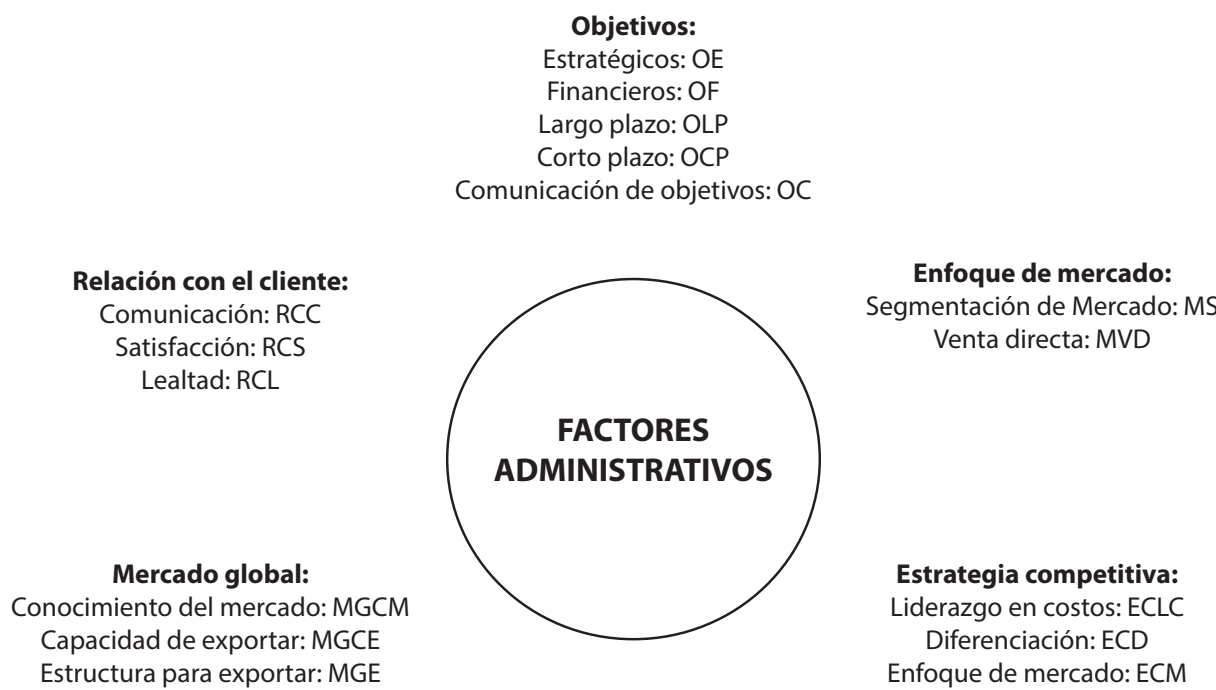

Fuente. Varela y Flores, (2014).

Los estudios mencionan la importancia de cada factor hacia la generación de innovación, aunque no señalan bajo qué condiciones se deben cumplir o qué criterios se deben aplicar en la ejecución. Por ello, es relevante hacer una investigación empírica para detectar los aspectos que se deben cubrir en cada factor y así plantear las hipótesis de investigación. A continuación, se hace un análisis de cada uno de los factores.

\subsubsection{Objetivos}

El proceso de innovación ha sido relacionado con características específicas, tales como que la organización debe contar con objetivos claros (Cormican y O'sullivan, 2004; Terziovski, 2003; Womack y Jones, 2005). En la investigación bibliográfica, se encuentra que las organizaciones se deben plantear dos tipos de objetivos para promover la innovación. Primero, los objetivos estratégicos que ayudan a las empresas a adquirir características que les sirvan para sobrevivir, prosperar y finalmente alcanzar sostenibilidad empresarial. Segundo, los objetivos financieros que ayudan a las empresas a tener una adecuada rentabilidad y fortaleza financiera (Fred, 2008; Kotler y Keller, 2006; Thompson y Gamble, 2012). El conjunto de objetivos financieros y estratégicos de una empresa debe proyectarse a corto y largo plazo. Los de corto plazo permiten ir midiendo el desempeño de la organización y los de largo plazo son cruciales para ir alcanzando un desempeño óptimo, pues permiten tener una visión en el futuro de lo que la empresa desea ser (Fred, 2008; Thompson y Gamble 
2012). Los objetivos para que sean claros deben ser publicados, y han de reafirmarse con frecuencia, esencialmente, a través de una comunicación efectiva (Daft, 2004).

Hipótesis 1a: existe una relación entre los objetivos estratégicos y financieros, objetivos a corto y largo plazo y comunicar los objetivos con la generación de innovación técnica en las PyMEs del ramo de las autopartes.

Hipótesis 1b: existe una relación entre los objetivos estratégicos y financieros, objetivos a corto y largo plazo, y comunicar los objetivos con la generación de innovación administrativa en las PyMEs del ramo de las autopartes.

\subsubsection{Enfoque de mercado}

La orientación al mercado como filosofía de negocio actúa como una generadora de innovación (Low y Purchase, 2005; Webb, Webster y Krepapa, 2000). Para que exista una orientación al mercado, debe existir una segmentación que es la selección de los sectores industriales en los que debe competir la empresa. Por tanto, si una empresa desea promover la innovación, es importante que tenga segmentado el mercado (Andersen y Buvik, 2002; Kotler, 2011). También la comercialización promueve la innovación desde la fuerza de ventas (Avlonitis, Papastathopoulou y Gounaris, 2001), por lo cual es fundamental la generación de venta de forma personal para que exista una comunicación efectiva con el cliente, ya que, a partir de la relación directa que se tiene con los consumidores, se proporcionan ideas que se pueden generar en innovación (Martín, Román y Fernández, 2011).

Hipótesis 2a: existe una relación entre tener un enfoque de mercado orientado a la segmentación y realizar una venta directa con el cliente con la generación de innova- ción técnica en las PyMEs del ramo de las autopartes.

Hipótesis 2b: existe una relación entre tener un enfoque de mercado orientado a la segmentación y realizar una venta directa con el cliente con la generación de innovación administrativa en las PyMEs del ramo de las autopartes.

\subsubsection{Estrategia competitiva}

Aquellas organizaciones que deseen promover la innovación deben tener una estrategia competitiva (LeskovarSpacapan y Bastic, 2007; Mu, Peng y Maclachlan, 2009); el liderazgo en costos, la diferenciación y el enfoque de mercado son estrategias que adoptan las empresas, y depende del desarrollo generar en mayor o menor medida algún tipo de innovación (Webb, Webster y Krepapa, 2000). Adoptar cualquiera de las estrategias competitivas implica de una $\mathrm{u}$ otra forma la realización de innovaciones (Daft, 2004; Kotler y Keller, 2006).

Hipótesis 3a: existe una relación entre tener una estrategia competitiva orientada a liderazgo en costos, una estrategia basada en la diferenciación o una estrategia con enfoque de mercado con la generación de innovación técnica en las PyMEs del ramo de las autopartes.

Hipótesis 3b: existe una relación entre tener una estrategia competitiva orientada a liderazgo en costos, una estrategia basada en la diferenciación o una estrategia con enfoque de mercado con la generación de innovación administrativa en las PyMEs del ramo de las autopartes.

\subsubsection{Mercado global}

En la globalización, aquellas empresas que compiten en el mercado global emplean la innovación como una herramienta para 
lograrla (Anlló, Bisang, Campi y Albornoz, 2009; Kalantaridis, 2004; Leonidou, 2004), y para ello se debe tener una disponibilidad de recursos adecuados tales como contar con el conocimiento, la capacidad y la estructura organizacional; estos recursos y orientación a la exportación pueden actuar como motor o freno de la expansión internacional. Por último, Escandón y Hurtado (2014) mencionan la importancia de la internacionalización de las PyMEs como fuente de innovación.

Hipótesis 4a: existe una relación entre tener un enfoque de mercado global basado en el conocimiento, la capacidad y la estructura organizacional con la generación de innovación técnica en las PyMEs del ramo de las autopartes.

Hipótesis 4b: existe una relación entre tener un enfoque de mercado global basado en el conocimiento, la capacidad y la estructura organizacional con la generación de innovación administrativa en las PyMEs del ramo de las autopartes.

\subsubsection{Relación con el cliente}

Hoy en día, las compañías innovadoras no se ven a sí mismas vendiendo productos, sino que se ven creando clientes leales, pues ellos son fuente importante de innovación; la relación que existe con los clientes es vital (Kotler, 2011; Hamel, 2012), así se siente considerado como miembro de la compañía y aporta ideas de innovación logrando una mayor lealtad de su parte; las empresas deben lanzar programas de beneficios de tal forma que los miembros - clientesse resistan a abandonar y perder los privilegios, con ello se genera una relación a largo plazo (Grinstein, 2008; Llonch y López, 2004; Puente y Cervilla, 2007). Con estas acciones, el cliente se sentirá satisfecho porque la empresa se centra no solo en proveerle un producto, sino que la misma aborda las preocupaciones y ayuda a resolver los problemas y conflictos que el cliente tenga (Chanllagalla y Venkatesh, 2008; Sheth y Sharma, 2008).

Hipótesis 5a: existe una relación entre mantener una relación con el cliente centrada en la comunicación, satisfacción y lealtad con la generación de innovación técnica en las PyMEs del ramo de las autopartes.

Hipótesis 5b: existe una relación entre mantener una relación con el cliente centrada en la comunicación, satisfacción y lealtad con la generación de innovación técnica en las PyMEs del ramo de las autopartes.

Cada uno de estos elementos analizados proporciona criterios que cada empresa debe aplicar en las operaciones para promover la innovación en las organizaciones. 


\section{Metodología}

$\mathrm{E}_{\text {que se siguió en el estudio, incluye el }}^{\text {n esta seción explica la metología }}$ enfoque, el alcance, la población meta de la investigación, la definición de las variables dependientes y la definición de las variables independientes, el instrumento de medición, la recolección de datos y la justificación de la prueba estadística.

El enfoque de la investigación es cuantitativo, ya que se siguió una recolección de datos para probar una hipótesis empleando una escala numérica en el instrumento de medición y el uso de una herramienta estadística, mientras que el alcance de la investigación es correlacional, dado que se mide la relación que existe entre las variables innovación técnica e innovación administrativa con las cinco variables de los factores administrativos.

De acuerdo con el directorio empresarial de la Secretaría de Desarrollo Económico del Estado de Tlaxcala (2011), el total de PyMEs del ramo de las autopartes ubicadas en el Estado son 12, las cuales se dedican a la fabricación de arneses eléctricos, partes plásticas e interiores automotores. Por ser una población reducida, se determinó que el estudio se aplicará al $100 \%$ de las PyMEs.

Se definieron como variables dependientes la innovación técnica y la innovación administrativa, y como variables independientes los cinco factores administrativos, ya que lo que lo que se quiere investigar es si estos últimos tienen una influencia sobre la innovación técnica y la innovación administrativa. Más adelante, se muestran los factores administrativos con cada indicador y la relación tanto con la innovación técnica como con la innovación administrativa, así como la relación con cada una de las hipótesis plateadas (Figura 2).

Para obtener los datos, se diseñó un cuestionario para medir el comportamiento de las variables dependientes: innovación técnica e innovación administrativa, y las variables independientes objetivos: enfoque de mercado, estrategia competitiva, mercado global y relación con el cliente. Se empleó una escala de Likert de la siguiente forma: i) totalmente en desacuerdo; ii) en; iii) de acuerdo; y iv) totalmente de acuerdo. Del cuestionario cuatro preguntas se centraron en la variable innovación técnica y cuatro preguntas en la variable innovación administrativa; para las variables objetivos, enfoque de mercado, estrategia competitiva, mercado global y relación con el cliente. En total veinticuatro preguntas.

El cuestionario se aplicó a las 12 PyMEs, el cual fue respondido por gerentes generales, gerentes de producción, gerentes de ventas y mandos medios de las áreas de producción, ventas y calidad. Se aplicaron 36 encuestas, de las cuales 12 fueron respondidas por gerentes y 24 por mandos medios, mediante una visita de manera personal al domicilio de cada empresa; el periodo de aplicación de la encuesta fue en los meses de agosto y noviembre de 2014 . 
Figura 2. Relación entre variables dependientes y variables independientes.

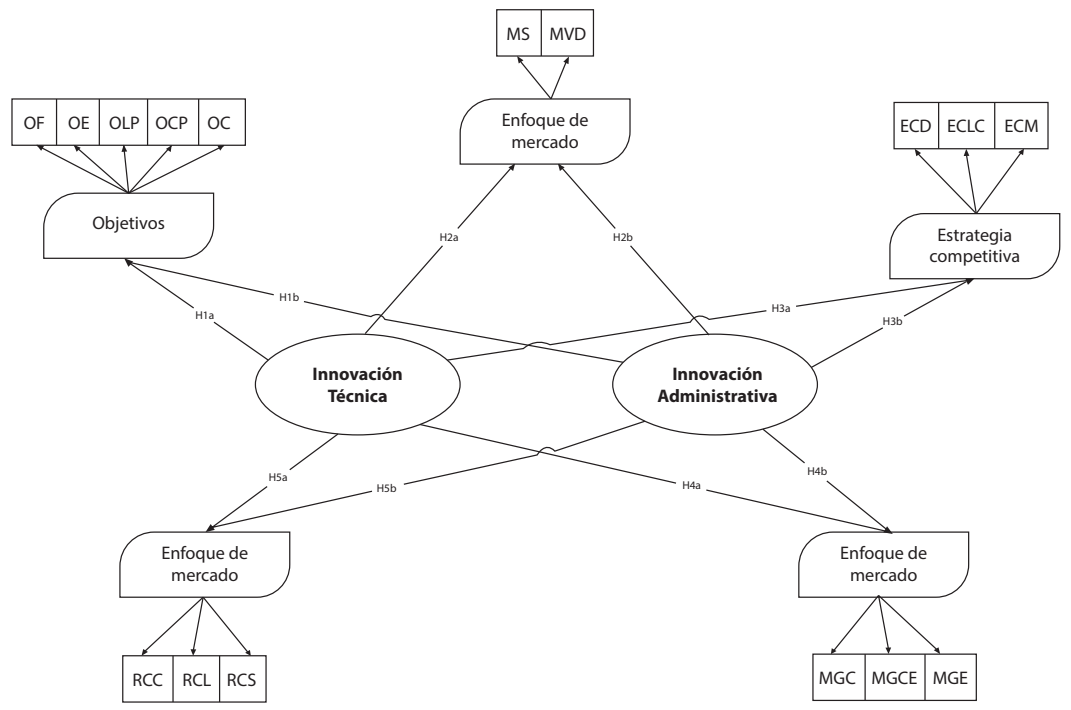

Fuente. Elaboración propia.

En cuanto a la confiabilidad del instrumento, se realizó la prueba de alfa de Cronbach que dio como resultado 0.947, lo cual significa que el instrumento es confiable. Se muestra el nivel de confiabilidad del instrumento (Tabla 2).

Tabla 2. Confiabilidad del instrumento

\begin{tabular}{|c|c|c|}
\hline Factores & Indicadores & a de Cronbach \\
\hline \multirow{2}{*}{ Innovación } & Técnica & 0.946 \\
\hline & Administrativa & 0.945 \\
\hline \multirow{5}{*}{ Objetivos } & Financieros & 0.949 \\
\hline & Estratégicos & 0.947 \\
\hline & Largo Plazo & 0.952 \\
\hline & Corto Plazo & 0.945 \\
\hline & Comunicación & 0.947 \\
\hline \multirow{2}{*}{ Enfoque del mercado } & Segmentación & 0.946 \\
\hline & Venta Directa & 0.947 \\
\hline \multirow{3}{*}{ Estrategia competitiva } & Diferenciación & 0.947 \\
\hline & Enfoque & 0.947 \\
\hline & Liderazgo en costos & 0.947 \\
\hline \multirow{3}{*}{ Mercado global } & Conocimiento del mercado extrangero & 0.947 \\
\hline & Capacidad para exportar & 0.945 \\
\hline & Estructura para exportar & 0.947 \\
\hline \multirow{3}{*}{ Relación con el cliente } & Comunicación & 0.945 \\
\hline & Lealtad & 0.946 \\
\hline & Satisfacción & 0.947 \\
\hline
\end{tabular}

Fuente. Elaboración propia. 
Para dar validez a las hipótesis planteadas, se realizó una prueba mediante el coeficiente de correlación rho de Spearman. Este ejercicio fue el adecuado, ya que los datos son no paramétricos, y además permite analizar la relación entre variables, una dependiente y una independiente, así como analizar el grado de influencia entre las variables. El modelo estadístico se conforma de la siguiente manera:

Innovación técnica $\mathrm{Y}_{1}=\beta_{1} \mathrm{OE}+\beta_{2} \mathrm{OF}+\beta_{3} \mathrm{OLP}+\beta_{4} \mathrm{OCP}+\beta_{5} \mathrm{OC}$

Innovación técnica

$$
\mathrm{Y}_{1}=\beta_{1} \mathrm{MS}+\beta_{2} \mathrm{MVD}
$$

Innovación técnica

$$
Y_{1}=\beta_{1} E C L C+\beta_{2} E C D+\beta_{3} E C M
$$

\section{Innovación técnica \\ $Y_{1}=\beta_{1} M G C M+\beta_{2}$ MGCE $+\beta_{3}$ MGE}

Innovación técnica

$$
Y_{1}=\beta_{1} R C C+\beta_{2} R C S+\beta_{3} R C L
$$

Innovación administrativa

$\mathrm{Y}_{2}=\beta_{1} \mathrm{OE}+\beta_{2} \mathrm{OF}+\beta_{3} \mathrm{OLP}+\beta_{4} \mathrm{OCP}+\beta_{5} \mathrm{OC}$

\section{Innovación administrativa}

$$
\mathrm{Y}_{12}=\beta_{1} \mathrm{MS}+\beta_{2} \mathrm{MVD}
$$

Innovación administrativa

$$
\mathrm{Y} 2=\beta 1 \mathrm{ECLC}+\beta 2 \mathrm{ECD}+\beta 3 \mathrm{ECM}
$$

Innovación administrativa

$$
\mathrm{Y}_{2}=\beta_{1} \mathrm{MGCM}+\beta_{2} \mathrm{MGCE}+\beta_{3} \mathrm{MGE}
$$

Innovación administrativa

$$
Y_{2}=\beta_{1} R C C+\beta_{2} R C S+\beta_{3} \text { RLC }
$$

\section{Resultados}

\begin{abstract}
A delante, se presentan los resultados de la correlación rho de Spearman que muestra la relación entre las variables innovación técnica e innovación administrativa con los cinco factores, y sitúa en juicio las hipótesis planteadas (Tabla 2). También se presenta el p-valor que da significancia a los datos $\mathrm{y}$ es indicador para aceptar o rechazar las hipótesis planteadas. Los resultados enseñan que existen más relaciones entre la innovación técnica con los factores: objetivos y relación con el cliente, mientras que existen
\end{abstract}

pocas relaciones entre innovación técnica y los factores: enfoque de mercado y estrategia competitiva, y es nula la relación entre innovación técnica con mercado global. En cuanto a la variable innovación administrativa, existen nulas relaciones con los factores: objetivos, enfoque de mercado, estrategia competitiva, mercado global y relación con el cliente, con lo cual se tendería a rechazar la hipótesis de que los factores administrativos influyen en la innovación administrativa. 
Tabla 3. Tabla de correlación rho de Spearman

\begin{tabular}{|c|c|c|c|c|c|c|c|c|c|}
\hline & & IT1 & IT2 & IT3 & IT4 & IA1 & IA2 & IA3 & IA4 \\
\hline OF & $\begin{array}{c}\text { correlación } \\
\text { p-valor }\end{array}$ & $\begin{array}{l}.353 \\
.056\end{array}$ & $\begin{array}{l}.299 \\
.108\end{array}$ & $\begin{array}{l}.295 \\
.113\end{array}$ & $\begin{array}{l}.327 \\
.078\end{array}$ & $\begin{array}{l}.225 \\
.232\end{array}$ & $\begin{array}{l}-.124 \\
.514\end{array}$ & $\begin{array}{l}.192 \\
.308\end{array}$ & $\begin{array}{l}-.058 \\
.759\end{array}$ \\
\hline $\mathrm{OE}$ & $\begin{array}{l}\text { correlación } \\
\text { p-valor }\end{array}$ & $\begin{array}{l}.454^{*} \\
.012\end{array}$ & $\begin{array}{l}.443^{*} \\
.014\end{array}$ & $\begin{array}{l}.492 * * \\
.0057\end{array}$ & $\begin{array}{l}.236 \\
.210\end{array}$ & $\begin{array}{l}-.108 \\
.571\end{array}$ & $\begin{array}{l}0.000 \\
1.000\end{array}$ & $\begin{array}{l}.449^{*} \\
.013\end{array}$ & $\begin{array}{l}-.260 \\
.165\end{array}$ \\
\hline OLP & $\begin{array}{c}\text { correlación } \\
\text { p-valor }\end{array}$ & $\begin{array}{l}.381 * \\
.038\end{array}$ & $\begin{array}{c}.479 * * \\
.007\end{array}$ & $\begin{array}{l}.721 * * \\
.000\end{array}$ & $\begin{array}{l}.516^{* *} \\
.0035\end{array}$ & $\begin{array}{l}-.201 \\
.287\end{array}$ & $\begin{array}{l}-.002 \\
.990\end{array}$ & $\begin{array}{c}.502^{* *} \\
.005\end{array}$ & $\begin{array}{l}-.044 \\
.817\end{array}$ \\
\hline OCP & $\begin{array}{c}\text { correlación } \\
\text { p-valor }\end{array}$ & $\begin{array}{c}.619 * * \\
.000\end{array}$ & $\begin{array}{l}-.347 \\
.060\end{array}$ & $\begin{array}{l}.406^{*} \\
.026\end{array}$ & $\begin{array}{l}.245 \\
.192\end{array}$ & $\begin{array}{l}-.143 \\
.451\end{array}$ & $\begin{array}{l}-.340 \\
.066\end{array}$ & $\begin{array}{l}.307 \\
.099\end{array}$ & $\begin{array}{l}-.303 \\
.104\end{array}$ \\
\hline OC1 & $\begin{array}{c}\text { correlación } \\
\text { p-valor }\end{array}$ & $\begin{array}{l}.054 \\
.778\end{array}$ & $\begin{array}{l}-0.37 \\
.846\end{array}$ & $\begin{array}{c}-.034^{*} \\
.859\end{array}$ & $\begin{array}{l}.132 \\
.487\end{array}$ & $\begin{array}{l}.051 \\
.789\end{array}$ & $\begin{array}{l}-.195 \\
.303\end{array}$ & $\begin{array}{l}.009 \\
.962\end{array}$ & $\begin{array}{l}.079 \\
.680\end{array}$ \\
\hline OC2 & $\begin{array}{c}\text { correlación } \\
\text { p-valor }\end{array}$ & $\begin{array}{l}.284 \\
.128\end{array}$ & $\begin{array}{l}.187 \\
.324\end{array}$ & $\begin{array}{l}.037 \\
.848\end{array}$ & $\begin{array}{l}.202 \\
.284\end{array}$ & $\begin{array}{l}.042 \\
.827\end{array}$ & $\begin{array}{l}-.053 \\
.780\end{array}$ & $\begin{array}{l}-.023 \\
.903\end{array}$ & $\begin{array}{l}-.028 \\
.885\end{array}$ \\
\hline MS1 & $\begin{array}{c}\text { correlación } \\
\text { p-valor }\end{array}$ & $\begin{array}{l}.171 \\
.367\end{array}$ & $\begin{array}{l}-.007 \\
.970\end{array}$ & $\begin{array}{l}.338 \\
.067\end{array}$ & $\begin{array}{l}.052 \\
.784\end{array}$ & $\begin{array}{l}.133 \\
.483\end{array}$ & $\begin{array}{l}-.168 \\
.373\end{array}$ & $\begin{array}{c}.499 * * \\
.005\end{array}$ & $\begin{array}{l}-.058 \\
.761\end{array}$ \\
\hline MS2 & $\begin{array}{c}\text { correlación } \\
\text { p-valor }\end{array}$ & $\begin{array}{l}-.059 \\
.757\end{array}$ & $\begin{array}{l}-.164 \\
.386\end{array}$ & $\begin{array}{l}.101 \\
.596\end{array}$ & $\begin{array}{l}.175 \\
.354\end{array}$ & $\begin{array}{l}.117 \\
.537\end{array}$ & $\begin{array}{l}.089 \\
.638\end{array}$ & $\begin{array}{l}.289 \\
.122\end{array}$ & $\begin{array}{l}.284 \\
.129\end{array}$ \\
\hline MVD1 & $\begin{array}{c}\text { correlación } \\
p \text {-valor }\end{array}$ & $\begin{array}{l}.122 \\
.521\end{array}$ & $\begin{array}{l}-.009 \\
.963\end{array}$ & $\begin{array}{l}-.019 \\
.921\end{array}$ & $\begin{array}{l}-.008 \\
.966\end{array}$ & $\begin{array}{l}.278 \\
.137\end{array}$ & $\begin{array}{l}.209 \\
.267\end{array}$ & $\begin{array}{l}.142 \\
.455\end{array}$ & $\begin{array}{l}.219 \\
.246\end{array}$ \\
\hline MVD2 & $\begin{array}{c}\text { correlación } \\
\text { p-valor }\end{array}$ & $\begin{array}{l}.216 \\
.252\end{array}$ & $\begin{array}{l}-.126 \\
.506\end{array}$ & $\begin{array}{l}-.116 \\
.542\end{array}$ & $\begin{array}{l}.184 \\
.331\end{array}$ & $\begin{array}{l}-.031 \\
.869\end{array}$ & $\begin{array}{l}-0.16 \\
.933\end{array}$ & $\begin{array}{l}-.053 \\
.781\end{array}$ & $\begin{array}{l}.127 \\
.504\end{array}$ \\
\hline ECD & $\begin{array}{c}\text { correlación } \\
\text { p-valor }\end{array}$ & $\begin{array}{l}.276 \\
.140\end{array}$ & $\begin{array}{l}.292 \\
.118\end{array}$ & $\begin{array}{c}.552^{* *} \\
.002\end{array}$ & $\begin{array}{l}.429 * \\
.018\end{array}$ & $\begin{array}{c}-.449 * \\
.013\end{array}$ & $\begin{array}{l}.049 \\
.797\end{array}$ & $\begin{array}{l}.204 \\
.279\end{array}$ & $\begin{array}{l}-.298 \\
.110\end{array}$ \\
\hline ECM1 & $\begin{array}{c}\text { correlación } \\
p \text {-valor }\end{array}$ & $\begin{array}{l}-.251 \\
.180\end{array}$ & $\begin{array}{l}-.107 \\
.575\end{array}$ & $\begin{array}{l}-.051 \\
-788\end{array}$ & $\begin{array}{l}-.089 \\
.639\end{array}$ & $\begin{array}{l}-.353 \\
.056\end{array}$ & $\begin{array}{l}-.109 \\
.567\end{array}$ & $\begin{array}{l}.142 \\
.455\end{array}$ & $\begin{array}{l}-.017 \\
.930\end{array}$ \\
\hline ECM2 & $\begin{array}{c}\text { correlación } \\
\text { p-valor }\end{array}$ & $\begin{array}{l}-.296 \\
.113\end{array}$ & $\begin{array}{l}-.293 \\
.116\end{array}$ & $\begin{array}{l}-.184 \\
.330\end{array}$ & $\begin{array}{l}-.127 \\
.504\end{array}$ & $\begin{array}{l}.363^{*} \\
.048\end{array}$ & $\begin{array}{l}-.063 \\
.741\end{array}$ & $\begin{array}{l}-.028 \\
.882\end{array}$ & $\begin{array}{l}.006 \\
.976\end{array}$ \\
\hline ECLC & $\begin{array}{c}\text { correlación } \\
\text { p-valor }\end{array}$ & $\begin{array}{l}.052 \\
.785\end{array}$ & $\begin{array}{l}-.047 \\
.805\end{array}$ & $\begin{array}{l}.022 \\
.908\end{array}$ & $\begin{array}{l}-.090 \\
.635\end{array}$ & $\begin{array}{l}.354 \\
.055\end{array}$ & $\begin{array}{l}.311 \\
.094\end{array}$ & $\begin{array}{l}.235 \\
.212\end{array}$ & $\begin{array}{l}.130 \\
.495\end{array}$ \\
\hline MGC1 & $\begin{array}{c}\text { correlación } \\
\text { p-valor }\end{array}$ & $\begin{array}{l}.314 \\
.092\end{array}$ & $\begin{array}{l}.241 \\
.199\end{array}$ & $\begin{array}{l}.330 \\
.075\end{array}$ & $\begin{array}{l}.356 \\
.053\end{array}$ & $\begin{array}{l}-.096 \\
.613\end{array}$ & $\begin{array}{l}-.157 \\
.408\end{array}$ & $\begin{array}{l}.212 \\
.260\end{array}$ & $\begin{array}{l}-.241 \\
.200\end{array}$ \\
\hline MGC2 & $\begin{array}{c}\text { correlación } \\
\text { p-valor }\end{array}$ & $\begin{array}{l}.478^{* *} \\
.008\end{array}$ & $\begin{array}{l}.638^{* *} \\
.000\end{array}$ & $\begin{array}{c}.493^{* *} \\
.006\end{array}$ & $\begin{array}{l}.668^{*} \\
.000\end{array}$ & $\begin{array}{l}.112 \\
.556\end{array}$ & $\begin{array}{l}.092 \\
.630\end{array}$ & $\begin{array}{l}.166 \\
.381\end{array}$ & $\begin{array}{l}.075 \\
.692\end{array}$ \\
\hline MGCE & $\begin{array}{c}\text { correlación } \\
\text { p-valor }\end{array}$ & $\begin{array}{c}.547^{* *} \\
.002\end{array}$ & $\begin{array}{c}.679 * * \\
.000\end{array}$ & $\begin{array}{l}.795 * * \\
.000\end{array}$ & $\begin{array}{l}.786 * * \\
.000\end{array}$ & $\begin{array}{l}-.196 \\
.300\end{array}$ & $\begin{array}{l}-.052 \\
.786\end{array}$ & $\begin{array}{c}.497^{* *} \\
.005\end{array}$ & $\begin{array}{l}-.125 \\
.511\end{array}$ \\
\hline MGE & $\begin{array}{c}\text { correlación } \\
\text { p-valor }\end{array}$ & $\begin{array}{c}.467^{* *} \\
.009\end{array}$ & $\begin{array}{c}.547^{* *} \\
.002\end{array}$ & $\begin{array}{c}.638^{* *} \\
.000\end{array}$ & $\begin{array}{c}.689 * * \\
.000\end{array}$ & $\begin{array}{l}-.355 \\
.054\end{array}$ & $\begin{array}{l}.153 \\
.420\end{array}$ & $\begin{array}{l}.412^{*} \\
.024\end{array}$ & $\begin{array}{l}-.145 \\
.444\end{array}$ \\
\hline RCC1 & $\begin{array}{c}\text { correlación } \\
\text { p-valor }\end{array}$ & $\begin{array}{l}.225 \\
.231\end{array}$ & $\begin{array}{l}.218 \\
.246\end{array}$ & $\begin{array}{l}.255 \\
.175\end{array}$ & $\begin{array}{l}.175 \\
.355\end{array}$ & $\begin{array}{l}-.281 \\
.133\end{array}$ & $\begin{array}{l}.267 \\
.154\end{array}$ & $\begin{array}{l}.427^{*} \\
.019\end{array}$ & $\begin{array}{l}.276 \\
.139\end{array}$ \\
\hline RCC2 & $\begin{array}{c}\text { correlación } \\
\text { p-valor }\end{array}$ & $\begin{array}{l}.084 \\
.657\end{array}$ & $\begin{array}{l}-.018 \\
.926\end{array}$ & $\begin{array}{l}0.98 \\
.607\end{array}$ & $\begin{array}{l}.268 \\
.152\end{array}$ & $\begin{array}{c}.387^{* *} \\
.035\end{array}$ & $\begin{array}{l}-.160 \\
.399\end{array}$ & $\begin{array}{l}.275 \\
.142\end{array}$ & $\begin{array}{l}-.243 \\
.195\end{array}$ \\
\hline RCL1 & $\begin{array}{c}\text { correlación } \\
\text { p-valor }\end{array}$ & $\begin{array}{c}.482 * * \\
.007\end{array}$ & $\begin{array}{l}.328 \\
.077\end{array}$ & $\begin{array}{l}.408^{*} \\
.025\end{array}$ & $\begin{array}{l}.378^{*} \\
.039\end{array}$ & $\begin{array}{c}-.568 * * \\
.001\end{array}$ & $\begin{array}{l}-.260 \\
.165\end{array}$ & $\begin{array}{l}.105 \\
.582\end{array}$ & $\begin{array}{l}-.294 \\
.115\end{array}$ \\
\hline $\mathrm{RCL2}$ & $\begin{array}{c}\text { correlación } \\
\text { p-valor }\end{array}$ & $\begin{array}{c}.482 * * \\
.007\end{array}$ & $\begin{array}{l}.328 \\
.077\end{array}$ & $\begin{array}{l}.408^{*} \\
.025\end{array}$ & $\begin{array}{l}.378^{*} \\
.039\end{array}$ & $\begin{array}{c}-.568^{* *} \\
.001\end{array}$ & $\begin{array}{l}-.260 \\
.165\end{array}$ & $\begin{array}{l}.105 \\
.582\end{array}$ & $\begin{array}{l}-.294 \\
.115\end{array}$ \\
\hline RCS1 & $\begin{array}{c}\text { correlación } \\
\text { p-valor }\end{array}$ & $\begin{array}{l}.459 * \\
.011\end{array}$ & $\begin{array}{l}.353 \\
.056\end{array}$ & $\begin{array}{c}.480 * * \\
.007\end{array}$ & $\begin{array}{l}.661 * * \\
.000\end{array}$ & $\begin{array}{l}-.267 \\
.153\end{array}$ & $\begin{array}{l}-.125 \\
.510\end{array}$ & $\begin{array}{l}.296 \\
.112\end{array}$ & $\begin{array}{l}-.283 \\
.130\end{array}$ \\
\hline RCS2 & $\begin{array}{c}\text { correlación } \\
\text { p-valor }\end{array}$ & $\begin{array}{c}.674 * * \\
.000\end{array}$ & $\begin{array}{l}.515^{* *} \\
.007\end{array}$ & $\begin{array}{l}.572^{* *} \\
.001\end{array}$ & $\begin{array}{l}.582 * * \\
.001\end{array}$ & $\begin{array}{c}-.635 * * \\
.000\end{array}$ & $\begin{array}{l}-.282 \\
.132\end{array}$ & $\begin{array}{l}.264 \\
.159\end{array}$ & $\begin{array}{l}-.355 \\
.054\end{array}$ \\
\hline
\end{tabular}

Fuente. Elaboración propia. 
Tomando como base los resultados, se da respuesta a las hipótesis planteadas. En la hipótesis $\mathrm{H} 1 \mathrm{a}$, se plantea que tener objetivos estratégicos y financieros a corto y largo plazo, y comunicarlos, influye en la generación de innovación técnica en las PyMEs del ramo de las autopartes. Por lo anterior, se concluye que los objetivos estratégicos producen cambios en los procesos, los métodos de trabajo y las nuevas tecnologías, ya que el p-valor es mejor de 0.05 y las correlaciones son $0.454,0.443$ y 0.492 , mientras que los objetivos a largo plazo influyen en la mejora de los procesos, los métodos de trabajo, así como en los cambios de tecnología y diseño de nuevos productos, dado que el valor de $p$ es menor de 0.05 con correlaciones de $0.381,0.479$, 0.721 y 0.516 . Los objetivos financieros a corto plazo y comunicación de estos no tienen influencia con la innovación técnica.

En la hipótesis $\mathrm{H} 1 \mathrm{~b}$, se concluye que los objetivos financieros, objetivos estratégicos a corto y largo plazo, y la comunicación de estos no influyen en la innovación administrativa.

En la hipótesis $\mathrm{H} 2 \mathrm{a}$, donde se plantea que tener un enfoque de mercado orientado a la segmentación y realizar una venta directa con el cliente influyen en la generación de innovación técnica en las PyMEs del ramo de las autopartes, se concluye que no existe una relación entre las necesidades del segmento del mercado con los cambios de procesos, cambios de métodos de trabajo y cambios de tecnología y la innovación técnica, ya que los valores de $p$ son mayores de 0.05 .

Para la hipótesis H2b, también se rechaza, puesto que, como lo muestran los datos, la relación es nula, por tanto, tener una segmentación de mercado y ventas directas no influye en la innovación administrativa en las PyMEs del ramo de las autopartes.
La hipótesis $\mathrm{H} 3 \mathrm{a}$ plantea que tener una estrategia competitiva orientada a liderazgo en costos, diferenciación y enfoque de mercado ayuda a la generación de innovación técnica en las PyMEs del ramo de las autopartes, solo se acepta el hecho de que la estrategia de diferenciación influye en los cambios de tecnología y diseño de productos con correlación de 0.552 y 0.429 y con un p-valor menor de 0.05 , por tanto, se acepta que la estrategia de diferenciación repercute en la innovación técnica en la PyMEs del ramo de las autopartes, mientras que la estrategia de liderazgo en costos y enfoque de mercado no tiene influencia.

En la hipótesis $\mathrm{H} 3 \mathrm{~b}$, tener una estrategia competitiva orientada a liderazgo en costos, de diferenciación y enfoque de mercado influye en la generación de innovación administrativa en las PyMEs del ramo de las autopartes, se rechaza porque no se encuentran correlaciones para aceptarla.

En la hipótesis $\mathrm{H} 4 \mathrm{a}$, se plantea que tener un enfoque de mercado global con el conocimiento del mercado, la capacidad y la estructura organizacional para exportar influye en la generación de innovación técnica en las PyMEs del ramo de las autopartes, se acepta tener la capacidad para exportar con una correlación de 0.478 , $0.638,0.493,0.668,0.547,0.679,0.795$ y 0.786 y un p-valor menor de 0.05 , además de tener una estructura para exportar con una correlación de $0.467,0.547,0.638$ y 0.689 y p-valor menor de 0.05 , que tienen efecto en la innovación técnica, ya que existe una relación entre los cambios de métodos de trabajo, de tecnología y de diseño de producto con la existencia de recursos para exportar, mientras que tener conocimiento del mercado exterior no influye en la innovación técnica.En la hipótesis H4b, hay evidencia para rechazarla y se concluye 
que tener un enfoque de mercado global orientado al conocimiento, la capacidad y la estructura organizacional no influye en la generación de innovación administrativa en las PyMEs del ramo de las autopartes.

Por último, la hipótesis H5a muestra varias correlaciones, se acepta que la satisfacción del cliente con correlaciones 0.459 , $0.480,0.661,0.674,0.515,0.572$ y 0.582 y p-valor menor de 0.05 , y lealtad del cliente con correlaciones $0.482,0.408$ y 0.378 con un p-valor menor de 0.05 ayudan a generar innovación técnica en las PyMEs del ramo de las autopartes, mientras que en la comunicación con el cliente no existe significancia en los datos. Con respecto a la hipótesis $\mathrm{H} 5 \mathrm{~b}$, los datos muestran que no hay correlaciones, por lo cual la innovación administrativa no es ayudada con el hecho de mantener una relación con el cliente centrada en la comunicación y la satisfacción en las PyMEs del ramo de las autopartes.

\section{Conclusiones}

Considerando que el objetivo de este estudio es determinar si existe una relación entre los factores administrativos y la innovación técnica e innovación administrativa, se sugiere que las PyMEs del ramo de las autopartes den mayor importancia a la implementación de cambios en los productos, servicios, procesos, métodos de producción y cambios en el diseño del producto, en lugar de cambios en la estructura organizacional, mejora en las funciones de trabajo, cambios en los puestos de trabajo y mejora en el modelo de negocios. De esta manera, es evidente que existe una prioridad hacia la innovación técnica que se ve influida por los cinco factores administrativos con indicadores muy específicos, no tanto así para la innovación administrativa. Este resultado estaría en concordancia con el trabajo de Damanpour (1996) quien plantea que la introducción de innovaciones técnicas es importante para la efectividad organizacional, mientras que la innovación administrativa provee solo un balance entre la estructura social y la estructura técnica de la organización; entonces, las PyMEs del ramo de las autopartes están más interesadas en los aspectos técnicos que en lo social.

Uno de los posibles motivos del porqué estas PyMEs tienen una orientación a la innovación técnica es que están más orientadas a la eficiencia de sus procesos que son rutinarios y poco complejos. Para lograrlo, recurren a los cambios en los procesos a fin de cumplir con las especificaciones de los diseños de los productos y la reducción de costos, por lo cual es importante encontrar estrategias de innovación que les ayude a esa disminución de costos; por ejemplo, manejar esquemas más esbeltos de diseño y producción. Este tipo de organizaciones no están diseñadas para lograr innovaciones complejas, sino para ser eficientes (Mintzberg, Buinn y Voyer, 1997), y como tal, cada uno de los factores administrativos tiene poca influencia en la generación de innovación técnica, y nula, en la innovación administrativa. Hamel (2012) menciona que este tipo de empresas no son fuentes de innovación porque su enfoque principal es la eficiencia y la productividad. 
También es de mencionar que al darle más importancia a la innovación técnica y nula importancia a la innovación administrativa es evidente que no existe como tal una cultura de innovación integral formada por ambos tipos de innovaciones, ya que, teniendo una cultura de la innovación integral donde existan tanto la innovación técnica como innovación administrativa, las empresas pueden plantear estrategias y modelos de negocio más novedosos (Gardini, Giuliani y Marricchi, 2011).

En cuanto a cada uno de los factores administrativos, se concluye que la aplicación coherente de los objetivos desempeña un papel central en la estrategia de innovación y en el desarrollo de las compañías, como bien lo menciona Simon (2009). Para motivar la innovación en las PyMEs del ramo de las autopartes, deben orientar sus objetivos de forma estratégica y ser a largo plazo, lo cual está en parte de acuerdo con lo mencionado por Fred (2008), Kotler y Keller (2006) y Thompson y Gamble (2012), puesto que los objetivos financieros a corto plazo y la comunicación de objetivos no influyen en la generación de innovación.

La segmentación de mercado debe estar bien definida por parte de las PyMEs, dado que ofrece las siguientes ventajas: las compañías pueden identificar más fácilmente a los clientes, convertirse en una opción para ese segmento y así obtener mayor margen en el mercado (Kotler, 2011). Esto coincide con lo mencionado por Andersen y Buvik (2002), que sustentan que la segmentación de mercado es importante para la generación de innovación. Sin embargo, de acuerdo con el estudio, no existe una relación directa con la innovación técnica y la innovación administrativa en las PyMEs del ramo de las autopartes.
Según los resultados, estas organizaciones deben buscar una estrategia de diferenciación, ya que orilla a las empresas a crear algo que sea percibido en el mercado como único, que sea diferente (Porter, 1990). Esto motiva a las empresas a diseñar productos y procesos que cumplan esta premisa a través de la innovación, en parte de acuerdo con lo que menciona Daft (2004), Simon (2009) y Skyrt y Antonic (2004), para quienes adoptar cualquier estrategia, ya sea de diferenciación, de liderazgo en costos y de enfoque de mercado, se realiza innovación.

Las PyMEs del ramo de las autopartes deben tener una orientación hacia el mercado exterior. Anlló et al. (2009) menciona que al exportar se requiere una gestión para construir un sistema que facilite el desarrollo de las innovaciones que necesite el mercado extranjero. A diferencia de lo que menciona Leonidou (2004) y Kalantaridis (2004), contar con la capacidad para exportar, que se traduce en tecnología, conocimientos y capacidad instalada, son factores para innovar, y esto equivale a contar con una estructura organizacional bien definida, no solo quedándose en el puro conocimiento del mercado.

Estas PyMEs han de implementar programas de beneficios para los clientes, de tal forma que los miembros - clientesse resistan a abandonar y perder los privilegios (Grinstein, 2008), lo cual les permitirá construir relaciones a largo plazo. Como bien lo mencionan Grinstein (2008) y Puente y Cervilla (2007), las empresas deben tener una orientación en mejorar la satisfacción de las necesidades de los clientes y así fidelizarlos, con esto incentivan la innovación, no basta con tener una comunicación con el cliente. 
La principal limitación de este estudio es el tamaño de la muestra - casos de PyMEs del ramo de las autopartes establecidas en el estado de Tlaxcala, México-. Lo anterior atenta contra la generalidad de los resultados obtenidos, además de que este estudio impide evidenciar el impacto que los aspectos de tecnología, oportunidades tecnológicas, concentración, intensidad de la competencia y la cultura organizacional tienen sobre la generación de innovación técnica e innovación administrativa. Otra limitante es que esta investigación impide observar la evolución de las variables a lo largo del tiempo, entendiendo que un estudio dinámico o longitudinal permite identificar variables y comportamientos que permanecen ocultos en los estudios estáticos. También el hecho de que la investigación se haya centrado en un sector específico no permite evaluar cómo es el comportamiento de las variables investigadas en otros sectores industriales y otro tamaño de empresa donde, sin duda, el comportamiento de las variables sea diferente e incluso esté con mayor sustento cada uno de los planteamientos generado por los autores consultados.
Los resultados de la presente investigación, a pesar de sus limitaciones, tienen importantes implicaciones para que las PyMEs pongan en operación aquellos factores administrativos que tienen influencia en la generación de innovación técnica, sin dejar de lado la innovación administrativa para dar el justo equilibrio entre lo técnico y social. Obviamente, se muestra la necesidad de incentivar la formación de PyMEs orientadas no solo ejecutar los factores administrativos, sino también a incentivar una innovación integral.

Los resultados de esta investigación se constituyen en puntos de partida para futuros estudios. Dado que los factores administrativos analizados no tienen influencia en la generación de innovación administrativa, sería interesante investigar sobre qué tipo de factores, y no solo administrativos sino de otra índole, sí tienen influencia, aunado a esto indagar si este tipo de factores se pueden aplicar en otro sector o en otro tipo de industria. 


\section{Referencias}

Adams, R., Bessant, J. y Phelps, R. (2006). Innovation management measurement: A review. International Journal of Management Reviews, 8(1), 21-47.

Afuah, A. (1999). La dinámica de la innovación organizacional: el nuevo concepto para lograr ventajas competitivas y rentabilidad. (s.c), Oxford University Press.

Andersen, O. y Buvik, A. (2002). Firms internationalization and alternative approaches to the international customer/market selection. International Business Review, 11, 347-363.

Anlló, G., Bisang, R., Campi, M. y Albornoz, I. (2009). Innovación y competitividad en tramas globales. Santiago de Chile: Naciones Unidas/Cepal.

Avlonitis, G. J., Papastathopoulou, P. G. y Gounaris, S. P. (2001). An empirically-based typology of product innovativeness for new financial services: Success and failure scenarios. Journal of Product Innovation Management, 18, 324-342.

Berson, Y., Oreg, S. y Dvir, T. (2008). CEO values, organizational culture and firm outcomes. Journal of Organizational Behavior, 29, 615-633.

Bowen, F. E., Rostami, M. y Steel, P. (2010). Timing is everything: A meta-analysis of the relationships between organizational performance and innovation. Journal of Business Research, 63, 11791185.

Cash, J. I., Earl, M. J. y Morison, R. (2008). Teaming up to crack innovation and enterprise integration. Harvard Business Review, 86(11), 90-100.

Cervilla, M. A. (2005). Excelencia operacional mediante la innovación y el mejoramiento continuo de los procesos: experiencias en la industria venezolana de autopartes. Academia Revista Latinoamericana de Administración, 34, 47-61.

Cooper, R. G. y Kleinschmidt, E. J. (1995). Benchmarking the firm's critical success factors in new product development. Journal of Product Innovation Management, 12, 374-391.

Cormican, K. y O'sullivan, D. (2004). Auditing best practice for effective product innovation management. Technovation, 24, 819-829.
Challagalla, G., Venkatesh, R. y Kohli, A. K. (2009). Proactive post-sales service: When and why does it pay off? Journal of Marketing, 73, 70-89.

Chan Kim, W. y Mauborgne, R. A. (2004). Innovación de valor la lógica estratégica del alto crecimiento. Harvard Business Review, 82(8), 113-122.

Daft, R. L. (2004). Administración (6. ${ }^{\mathrm{a}}$ ed.). México: Thompson.

Daft, R. L. (2007). Teoría y diseño organizacional (9. ${ }^{a}$ ed.). México: Cengage.

Damanpour, F. (1996). Organizational complexity and innovation: Developing and testing multiple contingency model. Management Science, 42(5), 693-16.

Damanpour, F. y Wischnevsky, J. D. (2006). Research on innovation in organizations: Distinguishing innovation-generating from innovation-adopting organizations. Journal of Engineering and Technology Management, 23, 269-291.

Damanpour, F., Szabat, K. A. y Evan, W. M. (1989). The relationship between types of innovation and organizational performance. Journal of Management Studies, 26(6), 587-602.

Donate, M. J. y Guadamillas, F. (2008). Gestión del conocimiento organizativo, innovación tecnológica y resultados: una investigación empírica. Investigaciones Europeas de Dirección y Economía de la Empresa, 14, 139-167.

Ernst, H. (2002). Success factors of new product development: A review of the empirical literature. International Journal of Management Reviews, 4, 1-40.

Escandón, D. y Hurtado, A. (2014). Factores que influyen en el desarrollo exportador de las PyMEs en Colombia. Estudios Gerenciales, 30, 172-183.

Foxona, T. J., Grossa, R., Chaseb, A., Howesb, J., Arnallc, A. y Anderson, D. (2005). UK innovation systems for new and renewable energy technologies: Drivers, barriers and systems failures. Energy Policy, 33, 2123-2137. 
Fred, D. R. (2008). Conceptos de administración estratégica (11. a ed.). México: Pearson.

Gardini, M., Giuliani, G. y Marricchi, M. (2011). Finding the right place to start change. McKinsey $\mathcal{E}$ Company. Recuperado de http://ricardofabricio. com/images/Finding_the_right_place_to_start_ change_-_alunos.pdf

González Alvarado, T. E. y Martin Granados, M. A. (2013). La innovación en entornos económicos pocos favorables: el sector autopartes mexicano. Estudios Gerenciales, 29, 167-176.

Grinstein, A. (2008). The relationships between market orientation and alternative strategic orientations: A meta-analysis. European Journal of Marketing, 42(2), 115-134.

Hamel, G. (2012). What matters now: How to win in a world of relentless change, ferocious competition, and unstoppable innovation. San Francisco: Jossey-Bass.

Hull, F. M. y Tidd, J. (2003). Service innovation. Londres: Imperial College Press.

Kalantaridis, C. (2004). Internationalization, strategic behaviour and the small firm: A comparative investigation. Journal of Small Business Management, 42(3), 245-262.

Kotler, P. (2011). El marketing según Kotler: cómo crear, ganar y dominar mercados. Madrid: Paidós.

Kotler, P. y Keller, K. L. (2006). Dirección de marketing (12. ${ }^{\mathrm{a}}$ ed.). México: Pearson.

Kotler, P. y Caslione, J. A. (2010). Caótica: administración $y$ marketing en tiempos de caos. Bogotá: Norma.

Laforet, S. (2008). Size, strategic, and market orientation affects on innovation. Journal of Business Research, 61(7), 753-764.

Lee, H. y Kelley, D. (2008). Building dynamic capabilities for innovation: An exploratory study of key management practices. $R \& D$ Management, 38, 155-168.

Leonidou, L. (2004). An analysis of the barriers hindering small business export development. Journal of Small Business Management, 42(3), 79302.
LeskovarSpacapan, G. y Bastic, M. (2007). Differences in organizations' innovation capability in transition economy: Internal aspect of the organizations' strategic orientation. Technovation, 27, 533546.

Lin, C.-Y. (2006). Influencing factors on the innovation in logistics technologies for logistics service providers in Taiwan. The Journal of American Academy of Business, 9, 257-263.

López, N., Montes, J. M., Prieto, J. y Vázquez, C. J. (2008). Análisis de la regularidad innovadora en la empresa industrial española. Revista Europea de Dirección y Economía de la Empresa, 17, 7-28.

Low, D. R. y Purchase, S. (2005). Market orientation, performance and the market environment: a study of Australian manufacturing SME's. En ANZMAC 2005 Conference: Broadening the Boundaries.

Llonch, J. y López, M. (2004). La relación entre la orientación al mercado y los resultados y el efecto moderador de la estrategia genérica en dicha relación. Cuadernos de Economía y Dirección de la Empresa, 21, 65-88.

Manual de Oslo (2007). Directrices para la recogida $e$ interpretación de información relativa a innovación. México: OCDE y Eurostat.

Martensen, A., Dahlgaard, J. J., Park-Dahlgaard, S. M. y Grønholdt, L. (2007). Measuring and diagnosing innovation excellence-simple contra advanced approaches: A Danish study. Measuring Business Excellence, 11(4), 51-65.

Martín Castejón, P. J., Román Nicolás, S. y Fernández Sabiote, E. (2011). Influencia de los comportamientos relacionales del vendedor individual sobre la satisfacción, confianza y lealtad del comprador en un contexto de PyMEs industriales. Investigaciones Europeas de Dirección y Economía de la Empresa, 7(3), 91-109.

Mintzberg, H., Buinn, B. J. y Voyer, J. (1997). El proceso estratégico. México: Prentice Hall.

Mu, J. F., Peng, G. y Maclachlan, D. L. (2009). Effect of risk management strategy on NPD performance. Technovation, 29, 170180.

Porter, M. E. (1990). The competitive advantage of nations. Harvard Business Review, 68 (2), 73-93. 
Puente, R. y Cervilla, M. A. (2007). Prácticas de la gerencia de relaciones con el cliente (CRM) en empresas venezolanas: un estudio de casos. Academia, Revista Latinoamericana de Administración, $39,1-28$.

Rivas, L. A. y Flores, B. (2007). La gestión del conocimiento en la industria automovílistica. Estudios Gerenciales, 102(23), 83-100.

Saunila, M. y Ukko, J. (2014). Intangible aspects of innovation capability in SMEs: Impacts of size and industry. Journal of Engeniering and Technology Management, 33, 32-46.

Secretaría de Desarrollo Económico (2011). Directorio industrial del estado de Tlaxcala. México: Secretaría de Desarrollo Económico.

Sheth, J. N. y Sharma, A. (2008). The impact of the product to service shift in industrial markets and the evolution of the sales organization. Industrial Marketing Management, 37, 260-269.

Simon, H. (2009). Hidden champions of the twenty-first century: Success strategies of unknown world market leaders. Nueva York: Springer.

Skarzynski, P. y Gibson, R. (2008). Innovation to the core: A blueprint for transforming the way your company innovates. Boston: Harvard Business Press.

Skrt, B. y Antoncic, B. (2004). Strategic planning and small firm growth: An empirical examination. Managing Global Transitions, 2(2), 107-122.

Smith, M., Busi, M., Ball, P. y Van der Meer, R. (2008). Factors influencing an organisation's ability to manage innovation: a structured literature review and conceptual model. International Journal of Innovation Management, 12(4), 655-676.
Suñe, A., Bravo, E., Mundet, J. y Herrera, L. (2012). Buenas prácticas de innovación: un estudio exploratorio de empresas tecnológicas en el sector audiovisual español. Investigaciones Europeas de Dirección y Economía de la Empresa, 18, 139-147.

Terziovski, M. (2003). The relationship between networking practices and business excellence. Measuring Business Excellence, 7, 7892.

Thompson, I. P. y Gamble, I. S. (2012). Administración estratégica, teoría y casos (18. ${ }^{\mathrm{a}} \mathrm{ed}$.). México: McGrawHill.

Vahs, D. (2003). Organisation Einführung in die Organisationstheorie und-praxis. Stuttgart: SchäfferPoeschel Verlag.

Varela, J. A. y Flores, E. (2014). Prácticas administrativas que promueven la innovación en las empresas. Universidad \& Empresa, 16(27), 81101.

Verhaeghe, A. y Kfir, R. (2002). Managing innovation in a knowledge intensive technology organization (KITO). R\&D Management, 32, 409-417.

Vicencio, A. (2007). La industria automotriz en México: antecedentes, situación y perspectiva. Contaduría y Administración, 221, 211-248.

Webb, D., Webster, C. y Krepapa, A. (2000). An exploration of the meaning and outcomes of a customer defined market orientation. Journal of Business Research, 48, 101-112.

Womack, J. y Jones, D. (2005). Lean thinking. Barcelona: Gestión 2000.

Yamakawa, P. y Ostos, J. (2011). Relación entre innovación organizacional y desempeño organizacional. Revista Universidad E Empresa, 21, 93-115. 